\title{
Contact angles, ordering, and solidification of liquid mercury in carbon nanotube cavities
}

\author{
A. Kutana and K. P. Giapis* \\ Division of Chemistry and Chemical Engineering, California Institute of Technology, Pasadena, California 91125, USA
}

(Received 1 August 2007; published 29 November 2007)

\begin{abstract}
Optimized model potentials for mercury-mercury and mercury-carbon interactions are used in molecular dynamics simulations to study wetting and solidification of liquid mercury encapsulated in single-walled carbon nanotubes. The contact angle of mercury in the nanotube cavity increases linearly with wall curvature. The solid-liquid transition becomes less well defined as nanotube diameter decreases, while the melting temperature drops exponentially. A concentric cylindrical-shell structure is predicted for solidified mercury in small $(20,20)$ nanotubes, while a polycrystalline structure appears in larger $(40,40)$ nanotubes.
\end{abstract}

DOI: 10.1103/PhysRevB.76.195444

PACS number(s): $65.80 .+\mathrm{n}, 02.70 . \mathrm{Ns}, 64.70 . \mathrm{Dv}$

Liquid mercury does not wet graphite spontaneously. Indeed, fresh mercury forms droplets on highly ordered pyrolytic graphite with a contact angle of $152.5^{\circ} .1$ Being equivalent to graphene scrolls, carbon nanotubes too cannot be wetted spontaneously by mercury, or for that matter, by any liquid metal with surface tension greater than $180 \mathrm{mN} / \mathrm{m}^{2}$ However, wetting and filling of the inner cavity of a carbon nanotube with mercury have been shown to occur as a result of electrowetting. ${ }^{3}$ Continuous columns of liquid mercury form inside carbon nanotubes with their open end immersed into a mercury droplet, following the application of a voltage exceeding a threshold value between the nanotube and the droplet. Once confined in a nanotube cavity, the wetting behavior of a nonwetting fluid becomes of fundamental and practical interest for nanofluidics. Furthermore, studies of freezing or melting transitions of metals under onedimensional confinement could lead to the discovery of new crystalline phases and possibly to the existence of a solidliquid critical point. ${ }^{4}$ New solid metal phases could lead to unexpected mechanical, electrical, magnetic, and catalytic properties.

Ordering and crystallization of materials in carbon nanotube cavities have attracted intense theoretical interest. New ice phases, including ordered ice nanotubes, were predicted to form by freezing water inside carbon nanotubes. ${ }^{5}$ Simulations of $\mathrm{CCl}_{4}$ in nanotube cavities showed liquid ordering in concentric layers, which solidify into two-dimensional hexagonal crystals unlike those observed for bulk crystallization. $^{6}$ Depending on the nanotube diameter, helical-strand, cylindrical-shell, and fcc structures were predicted for $\mathrm{Cu}$ and $\mathrm{Au}$ confined inside nanotube cavities. ${ }^{7,8}$ Experimental evidence for the existence of such new structures in nanotube cavities has been sparse. Using highresolution transmission electron microscopy, Fan et al. ${ }^{9} \mathrm{dem}-$ onstrated the formation of a double-strand helix of iodine atoms inside a $(10,10)$ single-walled nanotube (SWCNT). In slightly larger SWCNTs, Guan et al. ${ }^{10}$ found a new crystalline phase of iodine, in addition to a triple-strand helix. Using x-ray diffraction, Maniwa et al. ${ }^{11}$ studied water freezing in SWCNT bundles and found a new peak appearing in the diffraction spectrum at $235 \mathrm{~K}$, which was attributed to the formation of ordered ice nanotubes. In the absence of confinement, suspended gold nanowires in ultrahigh vacuum exhibited a multishell structure composed of coaxial tubes. ${ }^{12}$ As of this writing, helical-strand, cylindrical-shell, or other new crystalline phases of metals encapsulated in SWCNTs have not been demonstrated experimentally.

In this work, we report results of classical molecular dynamics (MD) simulations performed to predict the contact angles and ordering of liquid mercury inside large singlewalled carbon nanotubes. The presence of a solid wall induces ordering in the adjacent liquid, which is manifested in density oscillations that extend several molecular diameters into the bulk. Furthermore, we report on the solidification process of liquid $\mathrm{Hg}$ under confinement and the structural properties of the $\mathrm{Hg}$ nanowires thus formed. We employ the optimized $\mathrm{Hg}-\mathrm{Hg}$ and $\mathrm{Hg}-\mathrm{C}$ potentials, developed for simulating mercury imbibition in carbon nanotubes activated by electrowetting. ${ }^{13}$ For the former, we use an ab initio pairwise potential for the mercury dimer developed by Schwerdtfeger et al., ${ }^{14}$ scaled to match the density of liquid $\mathrm{Hg}$ at $300 \mathrm{~K}$. This empirically adjusted potential improved the description of multiple mercury properties as compared to standard Lennard-Jones (LJ) potentials and was deemed a better candidate for studying wetting effects. Coincidentally, although not specifically developed for describing the solid phase, this potential reproduces the bulk freezing point and the interatomic distance of solid mercury (3.0 ̊). However, it should be emphasized that this potential is not a rigorous expansion of the energy of the bulk phase in terms of $n$-body $(n>2)$ interactions. When many-body terms are included, it has been shown ${ }^{15}$ that the convergence of the energy is poor at interatomic distances $(\sim 3 \AA)$ typical of the bulk phase, because the contributions from three-, four-, or even six-body terms decrease very slowly with $n$. Thus, potentials that include three- or four-body corrections may not result in improvement while complicating the form of the potential. The mercury-carbon interaction was modeled by a pairwise LJ potential, with energy and distance parameters $\left(\varepsilon_{\mathrm{HgC}} / k_{B}\right.$ $=15.0 \mathrm{~K}, \sigma_{\mathrm{HgC}}=3.321 \AA$ ) optimized according to the procedure described in Ref. 16 to predict the experimental contact angle of a macroscopic mercury drop on graphite at $300 \mathrm{~K}$.

Using these potentials, we calculated the shape of a liquid mercury drop on graphite and the $\mathrm{Hg}$ density oscillations (ordering) near the solid surface. The drop consisted of 4000 $\mathrm{Hg}$ atoms and it was placed on a graphite surface formed by two graphene layers with lateral dimensions of 108 $\times 107 \AA^{2}$, separated by $3.354 \AA$. The atoms of the liquid were coupled to a $300 \mathrm{~K}$ thermostat, while the positions of 

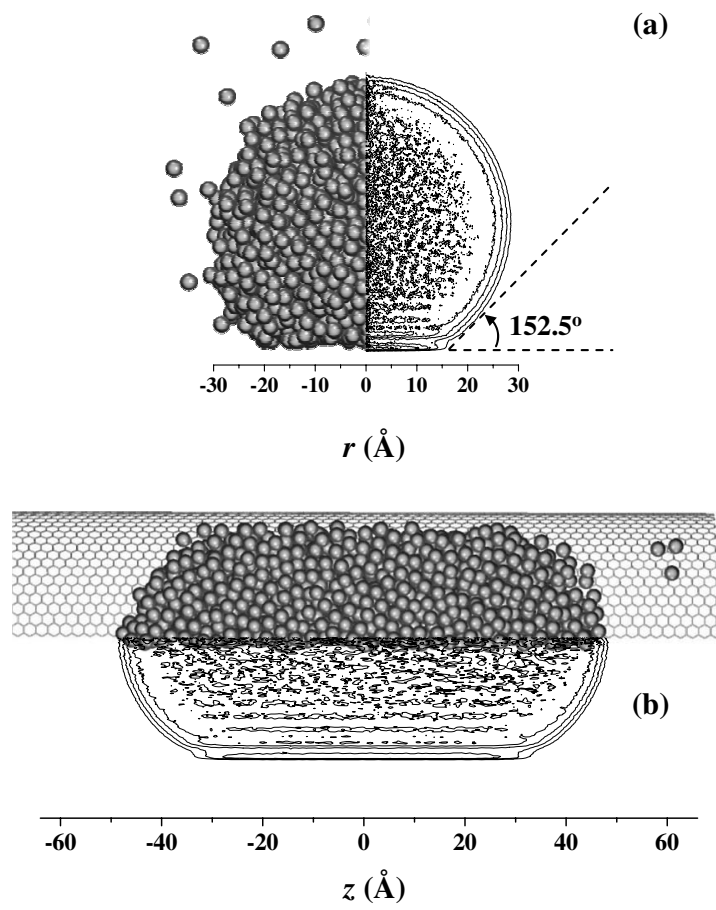

FIG. 1. (a) Snapshot of an equilibrated mercury drop (4000 atoms) on a graphene sheet (left) with averaged mercury density contour (right) with contact angle determination. (b) Snapshot of an equilibrated mercury slug (12 151 atoms) in a $(40,40)$ single-walled nanotube (top) with corresponding density contour (bottom). Note similarities in ordering of the liquid near the graphene sheet and nanotube walls.

the surface atoms were fixed. Evaporated mercury atoms were reflected toward the substrate to reproduce a saturated vapor ambient. Random position fluctuations of the drop center of mass were dampened mildly. The liquid density was recorded in a two-dimensional histogram as a function of the radial distance from the drop symmetry axis and height above the graphene plane. From this density, the position of the drop surface was determined assuming $\rho_{\text {surf }}$ $=(1 / 2) \rho_{\text {bulk }} \cdot{ }^{17}$ For smoothness, the recorded density function was averaged over a large time interval $(\sim 1 \mathrm{~ns})$ after a period of equilibration of the drop shape and vertical position. The equilibrium density map is shown in Fig. 1(a) along with a snapshot of the calculated drop shape where the $\mathrm{Hg}$ atoms are depicted as hard spheres. Ordering of mercury atoms adjacent to the graphene surface is apparent in the density map.

Next, the equilibrium wetting of mercury droplets inside carbon nanotubes is considered as a function of tube diameter. Different numbers of mercury atoms [(20,20), 1279; $(30,30), 3571 ;(40,40), 7123 ;(50,50), 12151]$ were placed in the cavity of single-walled $(n, n)$ nanotubes (SWCNT) at regular positions within a cylinder of radius $r=R_{\mathrm{SWCNT}}$ $-3.5 \AA$ and were equilibrated for at least 1000 ps at $300 \mathrm{~K}$ until a liquid slug was formed. Periodic boundary conditions along the nanotube axis ensured that evaporated $\mathrm{Hg}$ atoms did not escape. A Berendsen thermostat was applied to the fluid while the nanotube atoms were kept fixed. ${ }^{18}$ A snapshot of the MD simulations after 1190 ps of equilibration inside a

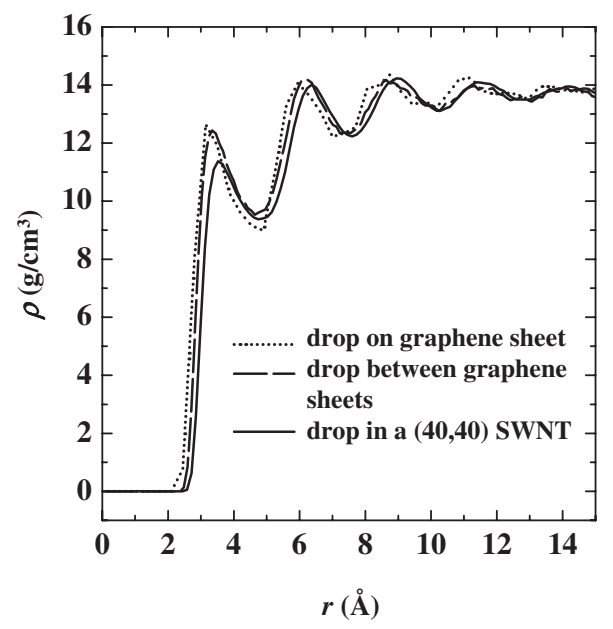

FIG. 2. Radial density profile of mercury as a function of the distance from a solid surface. Profiles are shown for a drop resting on a graphene sheet (dotted line), a drop confined between two graphene sheets separated by $54.24 \AA$ (dashed line), and a drop in a $(40,40)$ single-walled nanotube of diameter $=54.24 \AA$ (solid line).

$(40,40)$ SWCNT, shown in Fig. 1(b), reveals that the free surfaces of mercury are convex, as expected from a nonwetting interaction. The liquid density map is also shown in Fig. 1(b) as a function of the radial distance $r$ and axial length $z$. The atom ordering in the liquid near the curved nanotube walls is similar to that seen for the flat graphene surface. However, a comparison of the $\mathrm{Hg}$ density oscillations in Fig. 2 reveals a lower density value for the first layer nearest to the cavity walls, possibly due to the increased repulsion at the curved surface. A mercury drop confined between two parallel graphene sheets, separated by a distance of $54.24 \AA$ equal to the diameter of the $(40,40)$ SWCNT, exhibits identical density oscillations to the free drop on graphene, suggesting that confinement is less important for ordering than wall curvature. Indeed, the amplitude of the near-wall density oscillations grows with the nanotube wall curvature (not shown). The effect is well pronounced and is not likely to be artificially caused by the thermal motion of the nanotube walls. ${ }^{18}$ Instead, the stronger liquid ordering in smaller nanotubes may be caused by a hydrostatic capillary pressure $(P)$ that is proportional to the mean meniscus curvature $H$, as given by the Young-Laplace equation $\Delta P=2 H \gamma$, where $\gamma$ is the surface tension of the liquid in the drop. Enhanced ordering may occur when the spatial extent of the density oscillations becomes comparable with the tube radius. For example, the liquid is ordered throughout the tube volume in a $(20,20)$ SWCNT $(R=13.6 \AA)$.

The contact angle of the mercury free surface in the SWCNT cavity varies with nanotube diameter as a result of curvature. Figure 3(a) shows the meniscus outline and the corresponding fitting curve (circle) inside the $(30,30)$, $(40,40)$, and $(50,50)$ SWCNTs. Similarly to the flat surface, the contact angle $\theta$ was defined at the point of intersection of the fitting line with the liquid boundary in contact with the wall. The geometrical shape of the meniscus and the position of the liquid surface were found by fitting the contour lines corresponding to $\rho=(1 / 2) \rho_{\text {bulk }}$ with circular arcs. ${ }^{19}$ To ensure 

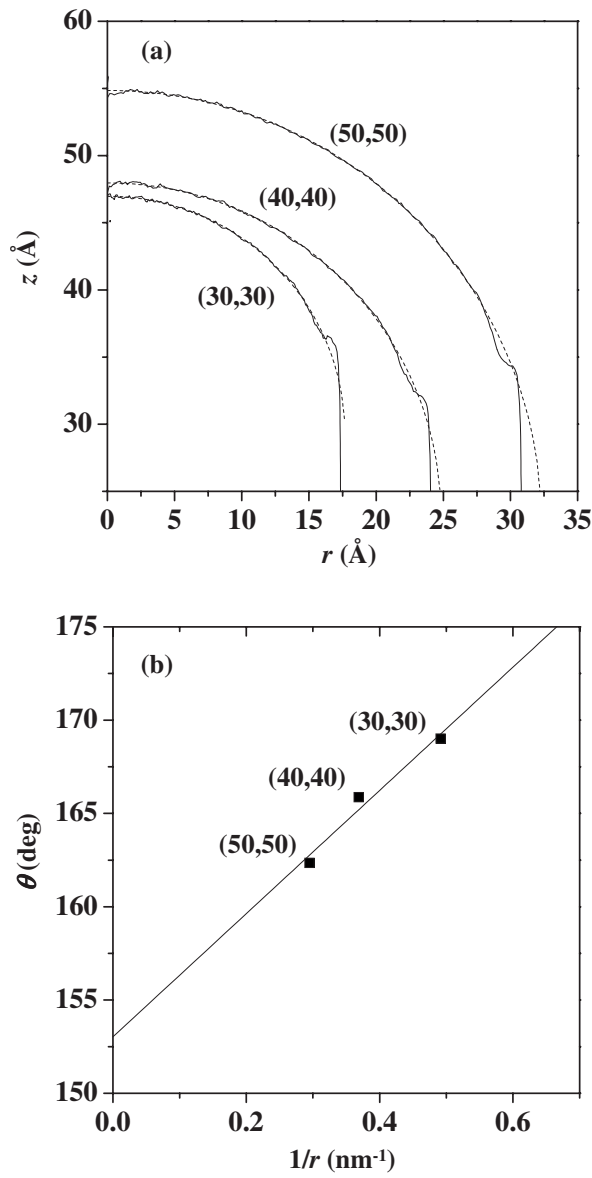

FIG. 3. (a) Outline of menisci for mercury slugs inside the indicated single-walled carbon nanotubes as a function of the distance from the tube centerline. Each shape is fitted with an arc (dotted line). (b) Mercury contact angles obtained from (a) as a function of nanotube curvature.

the smoothness of the contour lines, at least $5 \times 10^{5}$ samples of the liquid density were taken in calculating the average, and the points within $5 \AA$ from the wall and $2 \AA$ from the center axis were neglected in the fitting procedure.

Thus, obtained contact angles were larger than the $152.5^{\circ}$ value for a flat graphene sheet, suggesting a more mercurophobic nanotube interior. The contact angle increases linearly with the inverse of the tube radius, as inferred from Fig. 3(b), showing the contact angle of mercury inside $(30,30),(40,40)$, and $(50,50)$ SWCNTs. For smaller nanotubes $\left(R_{\text {SWCNT }}\right.$ $<17 \AA$ ), contact angles could not be calculated reliably because of the smallness of the fitting region. The cutoff angle of $153^{\circ}$, corresponding to a vanishing wall curvature, closely matches the result obtained for the drop on planar graphene..$^{20}$ This result confirms the importance of the wall curvature in determining the mercury contact angle in the nanotube cavity.

Remarkably, in one case of experimentally observed mercury encapsulated in a $(40,40)$ SWCNT, a contact angle of $150^{\circ} \pm 5^{\circ}$ was reported, ${ }^{3}$ with a meniscus resembling the curved surface of mercury in Fig. 1(b). This value is smaller than the contact angle of $\sim 166^{\circ}$, predicted for a $(40,40)$ SWCNT. A larger value for the nanotube cavity makes sense

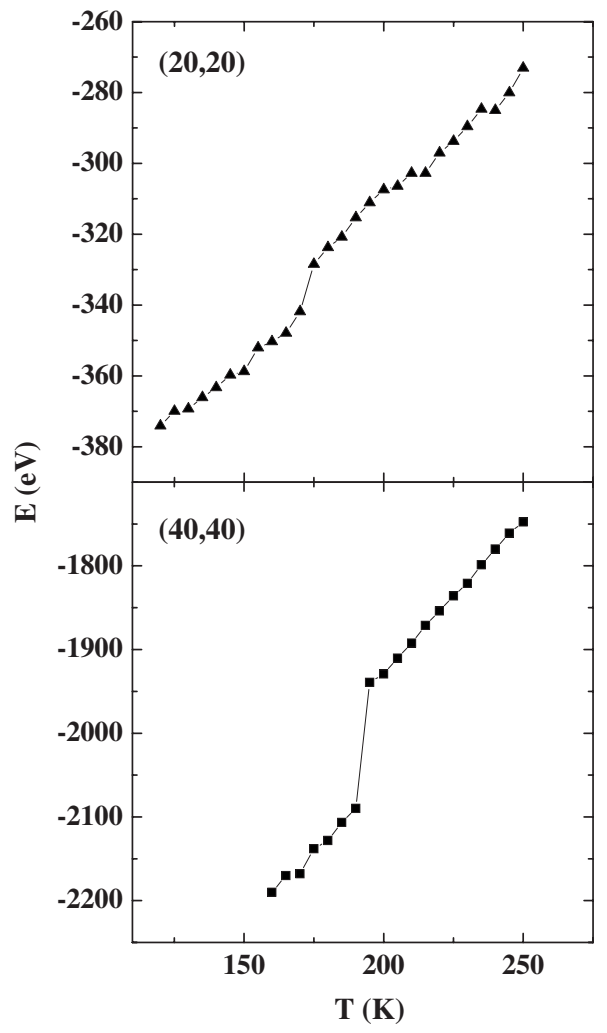

FIG. 4. Total energy of a mercury slug ( $7.9 \mathrm{~nm}$ long) encapsulated in the cavity of $(20,20)$ and $(40,40)$ single-walled nanotubes, calculated as a function of temperature after equilibration.

given the more mercurophobic character of the curved walls of the nanotube interior. Perhaps the smaller contact angle seen experimentally is a result of reduced surface tension because of oxidation or the presence of other impurities on the mercury surface. Indeed, a trace of the meniscus with lighter contrast was left behind when the mercury evaporated as a result of electron beam heating during imaging of the experimental profile. ${ }^{3}$

We next considered the solidification of mercury droplets encapsulated in SWCNT cavities. The internal energy of a droplet is determined as a function of temperature as follows. Each droplet is formed initially by joining two parts: onehalf liquid at $400 \mathrm{~K}$ and one-half solid at $50 \mathrm{~K}$. The number of atoms in each droplet varies with the nanotube diameter to keep the nanowire length fixed at $7.9 \mathrm{~nm}$ for all nanotube sizes studied. Then, the temperature is set to a desired value, and the system is allowed to equilibrate before calculating the total energy. The equilibration time increases substantially for the larger nanotubes (most runs were executed for 800 ps but a few points around the solidification temperature were allowed to equilibrate for $1600 \mathrm{ps})$. This procedure permits a more accurate determination of the solidification temperature by avoiding supercooling effects. Figure 4 illustrates results from calculations of the total energy of $\mathrm{Hg}$ droplets in $(20,20)$ and $(40,40)$ SWCNTs, plotted as a function of temperature. While the solid-liquid phase transition is readily apparent in both cases, it is less pronounced for the smaller nanotube. In fact, a mere change in the slope of the energy vs temperature plot indicates the phase transition for the $\mathrm{Hg}$ 


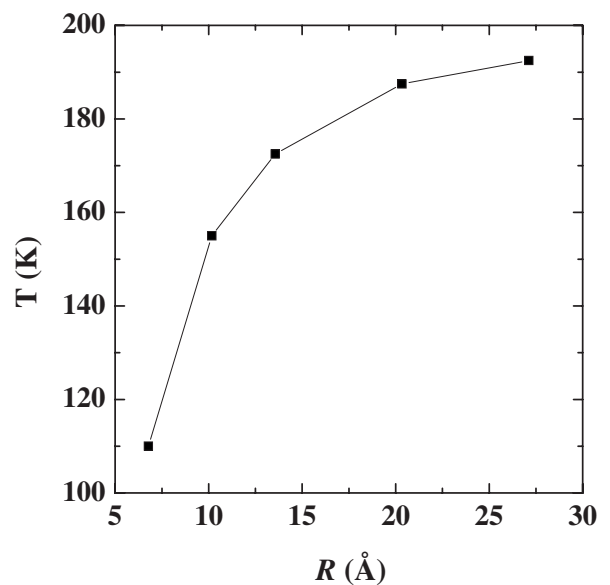

FIG. 5. Solidification temperature as a function of single-walled nanotube radius for a fixed-length mercury slug encapsulated in the nanotube.

slug in the $(10,10)$ SWCNT (not shown). This trend is consistent with the Landau fluctuation theorem as applied to phase transitions in reduced dimensionality systems. Figure 5 shows the solidification point for the fixed-length Hg nanowire in each nanotube as a function of nanotube radius. The melting transition occurs at exponentially lower temperatures as the nanotube size decreases. Note that the solidification point predicted for a macroscopic drop on graphite is $234 \mathrm{~K}$.

The cylindrical concentric shell structures predicted for solidified $\mathrm{Cu}$ and $\mathrm{Au}$ in SWCNTs, ${ }^{7,8}$ are also found for mercury in $(20,20)$ and smaller nanotubes. Figure 6(a) illustrates radial snapshots of the encapsulated mercury in a $(20,20)$ SWCNT at various temperatures above and below the solidification point $(\sim 173 \mathrm{~K})$. The $\mathrm{Hg}$ atoms are plotted as small hard spheres to facilitate observation. The first two snapshots correspond to a liquid phase with wall ordering, while the last two correspond to the solid phase. The radial density profile for each temperature, averaged over many snapshots, is shown in Fig. 6(b). Above the solidification point, the density oscillations have minima larger than zero, indicating motion of $\mathrm{Hg}$ atoms across layers and randomness characteristic of a liquid. However, below the solidification point, the density drops to zero between layers, suggesting that the $\mathrm{Hg}$ atoms are confined in each cylindrical shell. The lack of atom exchange between concentric shells occurs at the same temperature as the discontinuity in total energy and thus marks the solidification transition without ambiguity.

The density peaks become narrower in width and more pronounced at lower temperatures, indicating smaller fluctuations in atom positions in each shell. Detailed analysis of atom packing below the corresponding melting transition showed that the atoms in each shell assume a close-packed configuration. This packing gives the impression that the atoms organize in helices along the $60^{\circ}$ diagonal of the benzene rings of the carbon nanotube with respect to the tube axis, which could be interpreted as order imposed by the nanotube through templating. The lack of direct coordination between the $\mathrm{Hg}$ atoms and benzene rings suggests that templating would be an overinterpretation of close packing.

Increasing the nanotube diameter influences packing and
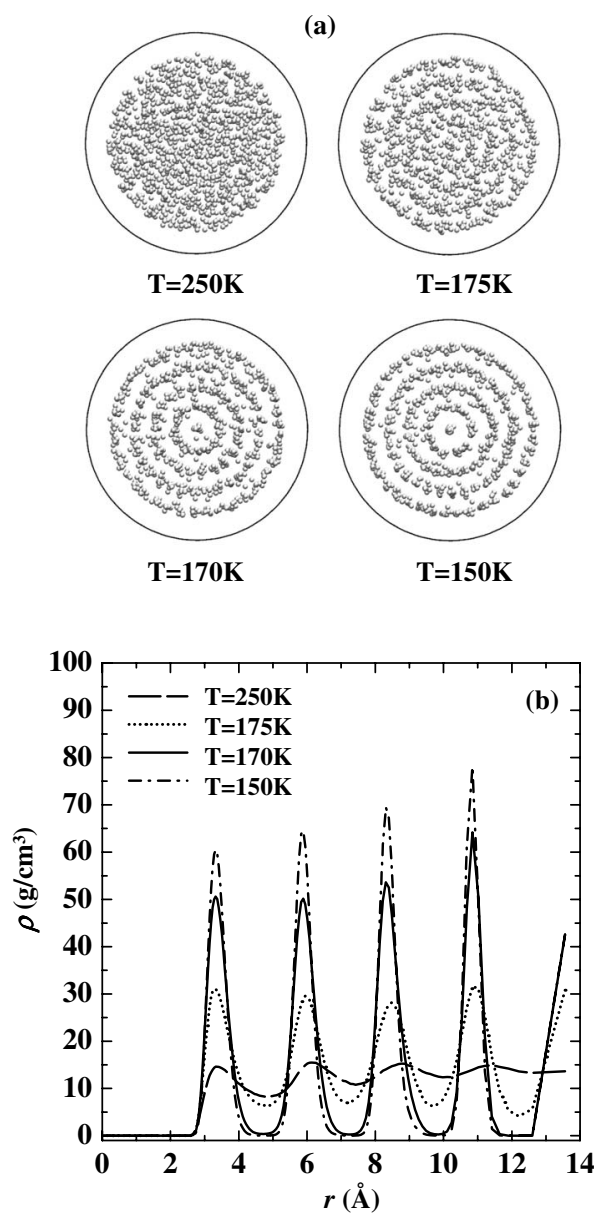

FIG. 6. (a) Front view of typical equilibrium configurations of encapsulated mercury in a $(20,20)$ single-walled nanotube at various temperatures above and below the solidification point $(\sim 173 \mathrm{~K})$. Only the centers of mass of the mercury atoms are depicted in the snapshots to facilitate observation. (b) Radial density profiles for the indicated temperatures, averaged over many snapshots.

ordering of the encapsulated mercury. Figure 7 presents snapshots for mercury in $(30,30)$ and $(40,40)$ SWCNTs at a select temperature below the corresponding solidification point. These solid structures must be contrasted with the concentric shells found in the $(20,20)$ SWCNT. While cylindrical-shell ordering persists near the walls, the order becomes much less discernible near the center of the $(30,30)$ SWCNT. Remarkably, the cylindrical-shell structure near the walls of the $(40,40)$ SWCNT changes into a different crystalline phase near the center axis. The mercury atoms organize in intersecting planar sheets parallel to the nanotube axis. We believe that cohesive interactions between mercury atoms become dominant away from the walls resulting in a bulklike ordering in the larger nanotubes. While new crystalline phases have been found for materials solidified in carbon nanotubes, a polycrystalline phase with a continuous transition from cylindrical order to a bulk phase has not been discussed. Such order should influence the structural and electronic properties of the solidified nanowire. Similar structures should also exist for encapsulated nanowires of 

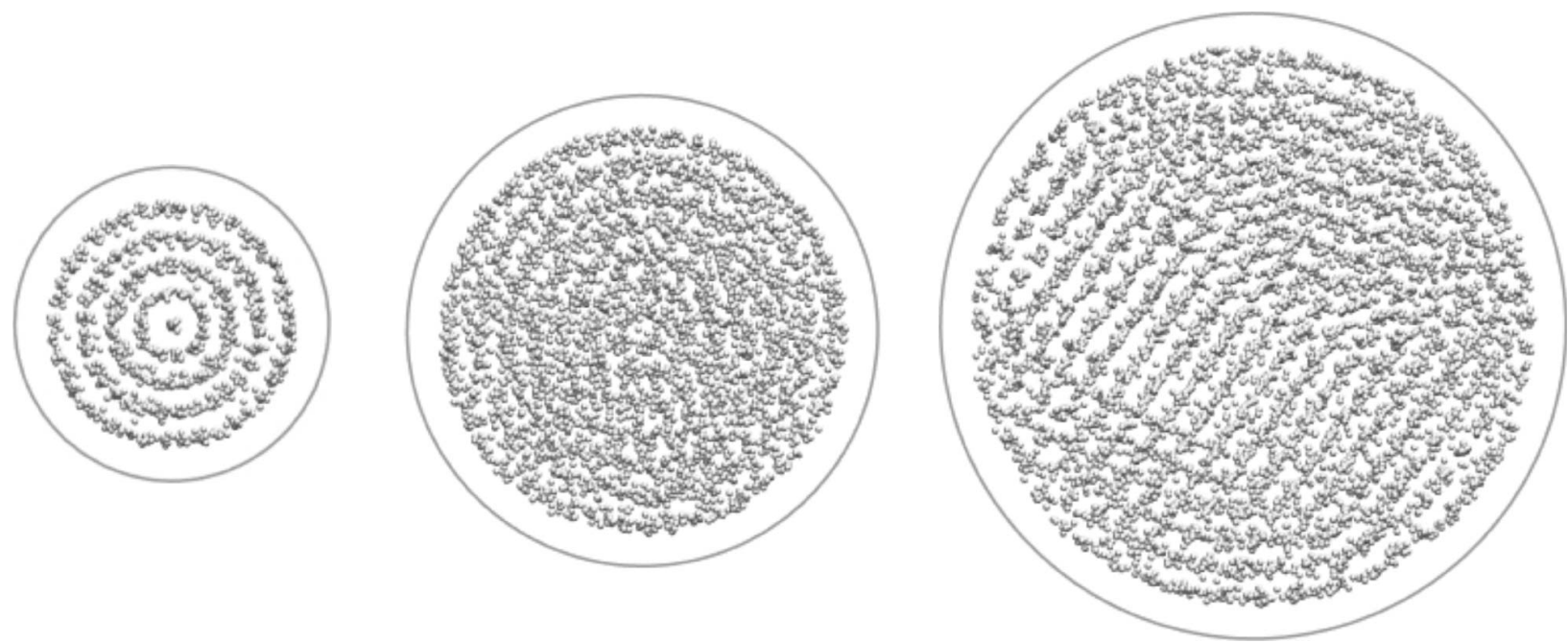

$(20,20) T=120 K$

$(30,30) T=150 K$

$(40,40) \mathrm{T}=160 \mathrm{~K}$

FIG. 7. Radial snapshots of solidified mercury, encapsulated in $(20,20),(30,30)$, and $(40,40)$ single-walled nanotubes at a representative temperature below the solidification point for each nanotube.

higher melting point metals, which may be easier than mercury to verify experimentally.

In conclusion, molecular dynamics simulations of liquid mercury encapsulated in carbon nanotube cavities predict ordering near the walls and density oscillations, which extend several molecular diameters into the bulk. The mercury wetting angle is found to increase linearly with wall curvature, suggesting that the nanotube interior is more mercurophobic than a flat graphene surface. Solidification of encapsulated mercury in small $(20,20)$ nanotubes results in close-packed concentric cylindrical-shell structures. In $(40,40)$ and larger nanotubes, the solidified mercury becomes polycrystalline: The cylindrical-shell order persists near the walls but evolves into a periodic planar structure away from the walls. The solid-liquid transition becomes less well defined as nanotube diameter decreases while the solidification temperature drops exponentially.

This work was based on research supported by NSF (CTS-0508096).
*Corresponding author; giapis@cheme.caltech.edu

${ }^{1}$ A. Awasthi, Y. J. Bhatt, and S. P. Garg, Meas. Sci. Technol. 7, 753 (1996).

${ }^{2}$ E. Dujardin, T. W. Ebbesen, H. Hiura, and K. Tanigaki, Science 265, 1850 (1994).

${ }^{3}$ J. Y. Chen, A. Kutana, C. P. Collier, and K. P. Giapis, Science 310, 1480 (2005).

${ }^{4}$ P. Ball, Nature (London) 361, 297 (1993).

${ }^{5}$ K. Koga, G. T. Gao, H. Tanaka, and X. C. Zeng, Nature (London) 412, 802 (2001).

${ }^{6}$ F. R. Hung, G. Dudziak, M. Sliwinska-Bartkowiak, and K. E. Gubbins, Mol. Phys. 102, 223 (2004).

${ }^{7}$ W. Y. Choi, J. W. Kang, and H. J. Hwang, Phys. Rev. B 68, 193405 (2003).

${ }^{8}$ S. Arcidiacono, J. H. Walther, D. Poulikakos, D. Passerone, and P. Koumoutsakos, Phys. Rev. Lett. 94, 105502 (2005).

${ }^{9}$ X. Fan, E. C. Dickey, P. C. Eklund, K. A. Williams, L. Grigorian, R. Buczko, S. T. Pantelides, and S. J. Pennycook, Phys. Rev. Lett. 84, 4621 (2000).

${ }^{10}$ L. Guan, K. Suenaga, Z. Shi, Z. Gu, and S. Iijima, Nano Lett. 7, 1532 (2007).

${ }^{11}$ Y. Maniwa, H. Kataura, M. Abe, S. Suzuki, Y. Achiba, H. Kira, and K. Matsuda, J. Phys. Soc. Jpn. 71, 2863 (2002).

${ }^{12}$ Y. Kondo and K. Takayanagi, Science 289, 606 (2000).

${ }^{13}$ A. Kutana and K. P. Giapis, Nano Lett. 6, 656 (2006).

${ }^{14}$ P. Schwerdtfeger, R. Wesendrup, G. E. Moyano, A. J. Sadlej, J. Greif, and F. J. Hensel, Chem. Phys. 115, 7401 (2001).

${ }^{15}$ B. Paulus, K. Rosciszewski, N. Gaston, P. Schwerdtfeger, and H. Stoll, Phys. Rev. B 70, 165106 (2004); N. Gaston and P. Schwerdtfeger, ibid. 74, 024105 (2006).

${ }^{16}$ T. Werder, J. H. Walther, R. L. Jaffe, T. Halicioglu, and P. Koumoutsakos, J. Phys. Chem. B 107, 1345 (2003); E. R. CruzChu, A. Aksimentiev, and K. Schulten, ibid. 110, 21497 (2006).

${ }^{17}$ J. S. Rowlinson and B. Widom, Molecular Theory of Capillarity (Clarendon, Oxford, 1982).

${ }^{18}$ Test runs with vibrating nanotube walls (at a nonzero temperature) yielded contact angles and density profiles virtually identical to those for fixed walls but at considerable computational cost.

${ }^{19}$ T. Werder, J. H. Walther, R. L. Jaffe, T. Halicioglu, F. Noca, and P. Koumoutsakos, Nano Lett. 1, 697 (2001).

${ }^{20}$ A separate simulation of a mercury droplet on a single graphene layer produced a contact angle identical to that for the two-layer case. 